\title{
On the classification of linear complementary dual codes
}

\author{
Makoto Araya*and Masaaki Harada ${ }^{\dagger}$
}

November 9, 2018

\begin{abstract}
We give a complete classification of binary linear complementary dual codes of lengths up to 13 and ternary linear complementary dual codes of lengths up to 10 .
\end{abstract}

\section{Introduction}

Let $\mathbb{F}_{q}$ denote the finite field of order $q$, where $q$ is a prime power. An $[n, k]$ code $C$ over $\mathbb{F}_{q}$ is a $k$-dimensional vector subspace of $\mathbb{F}_{q}^{n}$. A code over $\mathbb{F}_{2}$ is called binary and a code over $\mathbb{F}_{3}$ is called ternary. The parameters $n$ and $k$ are called the length and dimension of $C$, respectively. Two $[n, k]$ codes $C$ and $C^{\prime}$ over $\mathbb{F}_{q}$ are equivalent, denoted $C \cong C^{\prime}$, if there is an $n \times n$ monomial matrix $P$ over $\mathbb{F}_{q}$ with $C^{\prime}=C \cdot P=\{x P \mid x \in C\}$.

The dual code $C^{\perp}$ of an $[n, k]$ code $C$ over $\mathbb{F}_{q}$ is defined as $C^{\perp}=\left\{x \in \mathbb{F}_{q}^{n} \mid\right.$ $x \cdot y=0$ for all $y \in C\}$, where $x \cdot y$ is the standard inner product. A code $C$ is called linear complementary dual (or a linear code with complementary dual) if $C \cap C^{\perp}=\left\{\mathbf{0}_{n}\right\}$, where $\mathbf{0}_{n}$ denotes the zero vector of length $n$. We say that such a code is LCD for short. LCD codes were introduced by Massey [11] and gave an optimum linear coding solution for the two user binary adder channel. Recently, much work has been done concerning LCD codes for

${ }^{*}$ Department of Computer Science, Shizuoka University, Hamamatsu 432-8011, Japan. email: araya@inf.shizuoka.ac.jp

${ }^{\dagger}$ Research Center for Pure and Applied Mathematics, Graduate School of Information Sciences, Tohoku University, Sendai 980-8579, Japan. email: mharada@m.tohoku.ac.jp 
both theoretical and practical reasons (see [3], [4], [5], 8] and the references therein). In particular, we emphasize the recent work by Carlet, Mesnager, Tang, Qi and Pellikaan [4]. It has been shown in [4] that any code over $\mathbb{F}_{q}$ is equivalent to some LCD code for $q \geq 4$. This motivates us to study binary LCD codes and ternary LCD codes. In addition, recently, Carlet, Mesnager, Tang and Qi [3] have established the mass formulas. This motivates us to start a classification of binary LCD codes and ternary LCD codes. The aim of this note is to give a complete classification of binary linear complementary dual codes of lengths up to 13 and ternary linear complementary dual codes of lengths up to 10 .

The note is organized as follows. In Section 2, definitions, notations and basic results are given. We give some observation on the classification of binary LCD codes $C$ and ternary LCD codes $C$ with $d\left(C^{\perp}\right)=1$. Section 2 also presents the mass formulas given in [3] for binary LCD codes and ternary LCD codes. The mass formulas are an important role in the classification of binary LCD codes and ternary LCD codes. In Section 3, we give a complete classification of binary LCD $[n, k]$ codes and ternary LCD $[n, k]$ codes for $k=1, n-1$. In Section 4, we give a complete classification of binary LCD codes of lengths up to 13. In Section 5, we give a complete classification of ternary LCD codes of lengths up to 10.

\section{Preliminaries}

\subsection{Definitions, notations and basic results}

Let $C$ be an $[n, k]$ code over $\mathbb{F}_{q}$. The weight wt $(x)$ of a vector $x \in \mathbb{F}_{q}^{n}$ is the number of non-zero components of $x$. A vector of $C$ is called a codeword of $C$. The minimum non-zero weight of all codewords in $C$ is called the minimum weight $d(C)$ of $C$ and an $[n, k]$ code with minimum weight $d$ is called an $[n, k, d]$ code. The weight enumerator of $C$ is given by $\sum_{i=0}^{n} A_{i} y^{i}$, where $A_{i}$ is the number of codewords of weight $i$ in $C$. An automorphism of $C$ is an $n \times n$ monomial matrix $P$ over $\mathbb{F}_{q}$ with $C=C \cdot P$. The set consisting of all automorphisms of $C$ is called the automorphism group of $C$ and it is denoted by $\operatorname{Aut}(C)$. A generator matrix of $C$ is a $k \times n$ matrix whose rows are a set of basis vectors of $C$. A parity-check matrix of $C$ is a generator matrix of $C^{\perp}$.

Throughout this note, we use the following notations. Let $\mathbf{0}_{n}$ denote the 
zero vector of length $n$ and let $\mathbf{1}_{n}$ denote the all-one vector of length $n$. Let $I_{n}$ denote the identity matrix of order $n$ and let $A^{T}$ denote the transpose of a matrix $A$.

The following characterization is due to Massey [11].

Proposition 1. Let $C$ be a code over $\mathbb{F}_{q}$. Let $G$ and $H$ be a generator matrix and a parity-check matrix of $C$, respectively. Then the following properties are equivalent:

(i) $C$ is $L C D$,

(ii) $C^{\perp}$ is $L C D$,

(iii) $G G^{T}$ is nonsingular,

(iv) $H H^{T}$ is nonsingular.

The following proposition is trivial.

Proposition 2. Suppose that $C$ is an $L C D$ code over $\mathbb{F}_{q}$ and $q \in\{2,3\}$. If $C^{\prime}$ is equivalent to $C$, then $C^{\prime}$ is also $L C D$.

Throughout this note, we use the following notations. Let $\mathcal{B}_{n, k}$ denote the set of all inequivalent binary $\operatorname{LCD}[n, k]$ codes. Let $\mathcal{B}_{n, k, d}$ denote the set of all inequivalent binary $\operatorname{LCD}[n, k, d]$ codes. Let $\mathcal{C}_{n, k}$ denote the set of all inequivalent ternary $\operatorname{LCD}[n, k]$ codes. Let $\mathcal{C}_{n, k, d}$ denote the set of all inequivalent ternary $\operatorname{LCD}[n, k, d]$ codes.

\section{$2.2 \quad$ LCD codes $C$ with $d\left(C^{\perp}\right)=1$}

Let $C$ be an $[n, k, d]$ code over $\mathbb{F}_{q}$. Define the following $[n+1, k, d]$ code:

$$
C^{*}=\left\{\left(x_{1}, x_{2}, \ldots, x_{n}, 0\right) \mid\left(x_{1}, x_{2}, \ldots, x_{n}\right) \in C\right\} .
$$

Let $D$ be an $[n+1, k, d]$ code over $\mathbb{F}_{q}$ with $d\left(D^{\perp}\right)=1$. It is easy to see that there is an $[n, k, d]$ code $C$ over $\mathbb{F}_{q}$ with $D \cong C^{*}$. It is trivial that $C^{*}$ is LCD if and only if $C$ is LCD. In this way, every $\operatorname{LCD}[n+1, k, d]$ code $D$ over $\mathbb{F}_{q}$ with $d\left(D^{\perp}\right)=1$ is constructed from some $\operatorname{LCD}[n, k, d]$ code $C$ over $\mathbb{F}_{q}$. In addition, two LCD $[n+1, k, d]$ codes $D$ over $\mathbb{F}_{q}$ with $d\left(D^{\perp}\right)=1$ are equivalent if and only if two LCD $[n, k, d]$ codes $C$ over $\mathbb{F}_{q}$ are equivalent. Hence, all LCD $[n+1, k, d]$ codes $D$ over $\mathbb{F}_{q}$ with $d\left(D^{\perp}\right)=1$, which must be checked to achieve a complete classification, can be obtained from all inequivalent LCD $[n, k, d]$ codes $C$ over $\mathbb{F}_{q}$. Therefore, we have the following: 
Proposition 3. Let $\mathcal{B}_{n, k}^{*}$ denote the set of all inequivalent binary $L C D[n, k]$ codes $B$ with $d\left(B^{\perp}\right)=1$. Let $\mathcal{C}_{n, k}^{*}$ denote the set of all inequivalent ternary $L C D[n, k]$ codes $C$ with $d\left(C^{\perp}\right)=1$.

(i) There is a set $\mathcal{B}_{n-1, k}$ of all inequivalent binary $L C D[n-1, k]$ codes such that $\mathcal{B}_{n, k}^{*}=\left\{B^{*} \mid B \in \mathcal{B}_{n-1, k}\right\}$.

(ii) There is a set $\mathcal{C}_{n-1, k}$ of all inequivalent ternary $L C D[n-1, k]$ codes such that $\mathcal{C}_{n, k}^{*}=\left\{C^{*} \mid C \in \mathcal{C}_{n-1, k}\right\}$.

\subsection{Mass formulas}

It is trivial that $\left\{\mathbf{0}_{n}\right\}$ and $\mathbb{F}_{q}^{n}$ are the unique $\operatorname{LCD}[n, 1]$ code over $\mathbb{F}_{q}$ and the unique LCD $[n, n]$ code over $\mathbb{F}_{q}$, respectively. From now on, we assume that

$$
1 \leq k \leq n-1
$$

for an LCD $[n, k]$ code over $\mathbb{F}_{q}$.

Let $\mathcal{B}_{n, k}$ denote the set of all inequivalent binary LCD $[n, k]$ codes. Using the Gaussian binomial coefficients, the following values

$$
T_{2}(n, k)=\sum_{B \in \mathcal{B}_{n, k}} \frac{n !}{|\operatorname{Aut}(B)|}
$$

were determined theoretically in [3, Corollary 4.8], without finding the set $\mathcal{B}_{n, k}$, as follows:

$$
T_{2}(n, k)= \begin{cases}2^{\frac{n k-k^{2}+n-1}{2}}\left[\begin{array}{c}
\frac{n}{2}-1 \\
\frac{k-1}{2}
\end{array}\right]_{4} & \text { if } n \text { is even and } k \text { is odd, } \\
2^{\frac{(n-k)(k+1)}{2}\left[\begin{array}{c}
\frac{n-1}{2} \\
\frac{k-1}{2}
\end{array}\right]_{4}} & \text { if } n \text { and } k \text { are odd, } \\
2^{\frac{k(n-k+1)}{2}\left[\begin{array}{c}
\frac{n-1}{2} \\
\frac{k}{2}
\end{array}\right]_{4}} & \text { if } n \text { is odd and } k \text { is even, } \\
2^{\frac{k(n-k)}{2}}\left(2^{n-k}\left[\begin{array}{c}
\frac{n}{2}-1 \\
\frac{k}{2}-1
\end{array}\right]_{4}+\left[\begin{array}{c}
\frac{n}{2}-1 \\
\frac{k}{2}
\end{array}\right]_{4}\right) & \text { if } n \text { and } k \text { are even, }\end{cases}
$$

where

$$
\left[\begin{array}{l}
n \\
k
\end{array}\right]_{q}=\frac{\left(q^{n}-1\right)\left(q^{n-1}-1\right) \cdots\left(q^{n-k+1}-1\right)}{(q-1)\left(q^{2}-1\right) \cdots\left(q^{k}-1\right)} \text { if } k \neq 0,
$$

and $\left[\begin{array}{c}n \\ 0\end{array}\right]_{q}=1$. The value $T_{2}(n, k)$ is the number of the distinct binary LCD $[n, k]$ codes. 
Let $\mathcal{C}_{n, k}$ denote the set of all inequivalent ternary LCD $[n, k]$ codes. Similar to the above, the following values

$$
T_{3}(n, k)=\sum_{C \in \mathcal{C}_{n, k}} \frac{2^{n} n !}{|\operatorname{Aut}(C)|}
$$

were determined theoretically in [3, Corollary 5.9], without finding the set $\mathcal{C}_{n, k}$, as follows:

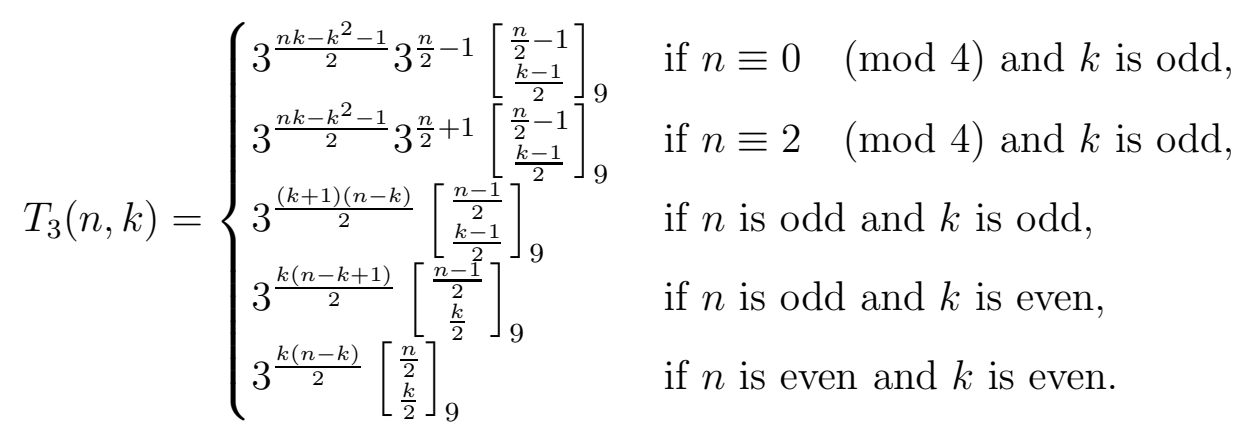

The value $T_{3}(n, k)$ is the number of the distinct ternary $\operatorname{LCD}[n, k]$ codes. The equations (21) and (3) are called the mass formulas.

\section{Classification for dimensions 1 and $n-1$}

In this section, we give a complete classification of binary LCD $[n, k]$ codes and ternary LCD $[n, k]$ codes for $k=1, n-1$.

Proposition 4. Let $\mathcal{B}_{n, k}$ denote the set of all inequivalent binary $L C D[n, k]$ codes. Let $\mathcal{B}_{n, k, d}$ denote the set of all inequivalent binary $L C D[n, k, d]$ codes.

(i) $\left|\mathcal{B}_{n, 1, d}\right|= \begin{cases}1 & \text { if } n \text { is even and } d=1,3,5, \ldots, n-1 \\ 1 & \text { if } n \text { is odd and } d=1,3,5, \ldots, n, \\ 0 & \text { otherwise. }\end{cases}$

(ii) $\left|\mathcal{B}_{n, 1}\right|=\left|\mathcal{B}_{n, n-1}\right|= \begin{cases}\frac{n}{2} & \text { if } n \text { is even, } \\ \frac{n+1}{2} & \text { if } n \text { is odd. }\end{cases}$

(iii) $\left|\mathcal{B}_{n, n-1, d}\right|= \begin{cases}\frac{n}{2} & \text { if } n \text { is even and } d=1, \\ \frac{n-1}{2} & \text { if } n \text { is odd and } d=1, \\ 1 & \text { if } n \text { is odd and } d=2, \\ 0 & \text { otherwise. }\end{cases}$ 
Proof. By considering a permutation of the coordinates, we may assume without loss of generality that a binary $[n, 1]$ code has generator matrix of the form $\left(\begin{array}{ll}1 & a\end{array}\right)$, where $a=\left(a_{1}, a_{2}, \ldots, a_{n-1}\right) \in \mathbb{F}_{2}^{n-1}$. We denote the code by $B(a)$. It is trivial that $B_{n}(a)$ is LCD if and only if $\operatorname{wt}(a)$ is even. If $\operatorname{wt}(a)=\operatorname{wt}(b)$, then it is trivial that $B_{n}(a)$ and $B_{n}(b)$ are equivalent. If wt $(a) \neq \operatorname{wt}(b)$, then $B_{n}(a)$ and $B_{n}(b)$ are inequivalent since $B_{n}(a)$ has weight enumerator $1+y^{1+\operatorname{wt}(a)}$. Hence, $B_{n}(a)$ and $B_{n}(b)$ are inequivalent if and only if $\operatorname{wt}(a) \neq \operatorname{wt}(b)$. The first two parts (i) and (ii) follow.

If $n$ is odd, then $B_{n}\left(\mathbf{1}_{n-1}\right)^{\perp}$ is the unique binary LCD $[n, n-1,2]$ code. If wt $(a)$ is even and $a \neq \mathbf{1}_{n-1}$, then $B_{n}(a)^{\perp}$ is a binary LCD $[n, n-1,1]$ code. The last part (iii) follows from (ii).

We give a complete classification of ternary $\operatorname{LCD}[n, k]$ codes for $k=$ $1, n-1$.

Proposition 5. Let $\mathcal{C}_{n, k}$ denote the set of all inequivalent ternary $L C D[n, k]$ codes. Let $\mathcal{C}_{n, k, d}$ denote the set of all inequivalent ternary $L C D[n, k, d]$ codes.

(i) $\left|\mathcal{C}_{n, 1, d}\right|= \begin{cases}1 & \text { if } d \not \equiv 0(\bmod 3), \\ 0 & \text { otherwise }\end{cases}$

(ii) $\left|\mathcal{C}_{n, 1}\right|=\left|\mathcal{C}_{n, n-1}\right|= \begin{cases}\frac{2 n}{3} & \text { if } n \equiv 0(\bmod 3), \\ \frac{2 n+1}{3} & \text { if } n \equiv 1(\bmod 3), \\ \frac{2 n+2}{3} & \text { if } n \equiv 2(\bmod 3) .\end{cases}$

(iii) $\left|\mathcal{C}_{n, n-1, d}\right|= \begin{cases}\frac{2 n}{3} & \text { if } n \equiv 0(\bmod 3) \text { and } d=1, \\ \frac{2 n+1}{3}-1 & \text { if } n \equiv 1(\bmod 3) \text { and } d=1, \\ 1 & \text { if } n \equiv 1(\bmod 3) \text { and } d=2, \\ \frac{2 n+2}{3}-1 & \text { if } n \equiv 2(\bmod 3) \text { and } d=1, \\ 1 & \text { if } n \equiv 2(\bmod 3) \text { and } d=2, \\ 0 & \text { otherwise. }\end{cases}$

Proof. By considering equivalent codes, we may assume without loss of generality that a ternary $[n, 1]$ code has generator matrix of the form ( $\left.\begin{array}{cc}1 & a\end{array}\right)$, where $a=\left(a_{1}, a_{2}, \ldots, a_{n-1}\right) \in \mathbb{F}_{3}^{n-1}$. We denote this code by $C_{n}(a)$. It is trivial that $C_{n}(a)$ is LCD if and only if $\operatorname{wt}(a) \not \equiv 2(\bmod 3)$. If $\operatorname{wt}(a)=\operatorname{wt}(b)$, then it is trivial that $C_{n}(a)$ and $C_{n}(b)$ are equivalent. If wt $(a) \neq \operatorname{wt}(b)$, then $C_{n}(a)$ 
and $C_{n}(b)$ are inequivalent since $C_{n}(a)$ has weight enumerator $1+2 y^{1+\mathrm{wt}(a)}$. Hence, $C_{n}(a)$ and $C_{n}(b)$ are inequivalent if and only if $\operatorname{wt}(a) \neq \operatorname{wt}(b)$. The first two parts (i) and (ii) follow.

If $n \not \equiv 0(\bmod 3)$, then $C_{n}\left(\mathbf{1}_{n-1}\right)^{\perp}$ is the unique ternary $\operatorname{LCD}[n, n-1,2]$ code. If $\operatorname{wt}(a) \not \equiv 2(\bmod 3)$ and $a \neq \mathbf{1}_{n-1}$, then $C_{n}(a)^{\perp}$ is a ternary LCD $[n, n-1,1]$ code. The last part (iii) follows from (ii).

\section{Classification of binary LCD codes of lengths up to 13}

In this section, we give a complete classification of binary LCD codes of lengths up to 13 . It is sufficient to consider $k \leq n / 2$, since the dual code of a binary LCD code is also LCD.

We describe how to complete a classification of binary LCD codes of lengths up to 13 . Every binary LCD $[n, k]$ code is equivalent to a binary code with generator matrix of the form $\left(\begin{array}{ll}I_{k} & A\end{array}\right)$, where $A$ is a $k \times(n-k)$ matrix. The set of matrices $A$ was constructed, row by row. Permuting the rows and columns of $A$ gives rise to different generator matrices which generate equivalent binary codes. Here, we consider some natural (lexicographical) order $<$ on the set of vectors of length $n-k$. We consider only matrices $A$, satisfying the condition $r_{1} \leq r_{2} \leq \cdots \leq r_{k}$, where $r_{i}$ is the $i$-th row of $A$. It is obvious that all binary codes, which must be checked to achieve a complete classification, can be obtained. By this method, we found all distinct binary LCD $[n, k]$ codes, which must be checked to achieve a complete classification for $2 \leq k \leq n / 2 \leq 11 / 2$. By determining the equivalence or inequivalence for a given pair of binary codes, we obtained the set $\mathcal{B}_{n, k}$ of all inequivalent binary LCD $[n, k]$ codes. The mass formula (2) shows that there is no other binary LCD $[n, k]$ code. This computation was performed in MAGma [2]. In principle, such a computation can be done by classifying binary LCD codes by the MAGMA function IsIsomorphic, then their automorphism groups can be calculated by AutomorphismGroup.

For $n=12$ and 13, the mass formula (2) was used to complete the classification, due to the computational complexity. Let $\mathcal{B}_{n, k}^{*}$ denote the set of all inequivalent binary LCD $[n, k]$ codes $B$ with $d\left(B^{\perp}\right)=1$. By Proposition 3 , there is a set $\mathcal{B}_{n-1, k}$ of all inequivalent binary LCD $[n-1, k]$ codes such that $\mathcal{B}_{n, k}^{*}=\left\{B^{*} \mid B \in \mathcal{B}_{n-1, k}\right\}$, where the construction of $B^{*}$ is listed in (1). Note 
that $\mathcal{B}_{n, n / 2}^{*}$ is constructed by considering the dual codes of the all inequivalent binary LCD $[n-1, n / 2-1]$ codes for the case $n=2 k$. Hence, it is sufficient to find all binary LCD $[n, k]$ codes $B$ with $d\left(B^{\perp}\right) \geq 2$, which must be checked further for equivalences. For these lengths, the set of matrices $A$ was constructed, column by column. Here, we consider some natural (lexicographical) order $<$ on the set of non-zero vectors of length $k$. We consider only matrices $A$, satisfying the condition $c_{1} \leq c_{2} \leq \cdots \leq c_{n-k}$, where $c_{i}$ is the $i$-th column of $A$. In this way, by adding new binary LCD codes, we continued to construct the set $\mathcal{B}_{n, k}^{\prime}$ of inequivalent binary LCD $[n, k]$ codes until $\sum_{B \in \mathcal{B}_{n, k}^{\prime}} \frac{n !}{|\operatorname{Aut}(B)|}$ reaches the value $T_{2}(n, k)$. When $\sum_{B \in \mathcal{B}_{n, k}^{\prime}} \frac{n !}{|\operatorname{Aut}(B)|}$ reaches the value $T_{2}(n, k)$, the classification was completed and $\mathcal{B}_{n, k}$ was obtained.

As a check, in order to verify that $\mathcal{B}_{n, k}$ contains no pair of equivalent binary LCD $[n, k]$ codes and that $\sum_{B \in \mathcal{B}_{n, k}} \frac{n !}{|\operatorname{Aut}(B)|}=T_{2}(n, k)$, we employed the package GUAVA [1] of GAP [6]. This calculation was done by using the functions IsEquivalent and AutomorphismGroup.

In order to illustrate our approach, we consider the case $(n, k)=(6,3)$ as an example. Let $B_{6,3, i}(i=1,2, \ldots, 8)$ be the binary LCD $[6,3]$ codes with generator matrices $\left(\begin{array}{ll}I_{3} & M_{i}\end{array}\right)$, where $M_{i}$ are listed in Table 1. We verified that these binary codes are inequivalent. Indeed, the binary codes $B_{6,3, i}$ have weight enumerators $W_{i}$, where

$$
\begin{array}{ll}
W_{1}=1+3 y^{2}+y^{3}+3 y^{5}, & W_{2}=1+3 y^{2}+3 y^{3}+y^{5}, \\
W_{3}=1+y^{2}+3 y^{3}+2 y^{4}+y^{5}, & W_{4}=1+3 y+3 y^{2}+y^{3}, \\
W_{5}=1+y+3 y^{2}+3 y^{3}, & W_{6}=1+y+y^{2}+y^{3}+2 y^{4}+2 y^{5}, \\
W_{7}=1+2 y+y^{2}+y^{3}+2 y^{4}+y^{5}, & W_{8}=1+y+y^{2}+3 y^{3}+2 y^{4} .
\end{array}
$$

This shows also that these binary codes are inequivalent. Since

$$
\left|\operatorname{Aut}\left(B_{6,3, i}\right)\right|=36,12,4,36,12,12,12,4(i=1,2, \ldots, 8),
$$

we have

$$
\sum_{i=1}^{8} \frac{6 !}{\left|\operatorname{Aut}\left(B_{6,3, i}\right)\right|}=640=T_{2}(6,3) .
$$

The mass formula (2) shows that there is no other binary LCD [6,3] code.

For $2 \leq k \leq n / 2$ and $n \leq 13$, we list in Table 6 the numbers $N$ of all inequivalent binary LCD $[n, k]$ codes and and the numbers $N_{d}$ of all inequivalent binary LCD $[n, k, d]$ codes. We also list the numbers $N_{d^{\perp}}$ of the dual $[n, n-$ 
Table 1: LCD $[6,3]$ codes

\begin{tabular}{c|c|c|c|c|c|c|c}
\hline$i$ & $M_{i}$ & $i$ & $M_{i}$ & $i$ & $M_{i}$ & $i$ & $M_{i}$ \\
\hline \multirow{2}{*}{1} & $\left(\begin{array}{l}001 \\
001 \\
110\end{array}\right)$ & 2 & $\left(\begin{array}{c}001 \\
001 \\
011\end{array}\right)$ & 3 & $\left(\begin{array}{c}001 \\
110 \\
111\end{array}\right)$ & 4 & $\left(\begin{array}{c}000 \\
000 \\
000\end{array}\right)$ \\
& $\left(\begin{array}{c}001 \\
001 \\
5\end{array}\right)$ & 6 & $\left(\begin{array}{c}001 \\
111 \\
000\end{array}\right)$ & 7 & $\left(\begin{array}{c}110 \\
000 \\
000\end{array}\right)$ & 8 & $\left(\begin{array}{c}001 \\
011 \\
000\end{array}\right)$ \\
\hline
\end{tabular}

Table 2: Smallest automorphism groups

\begin{tabular}{c|c|c|c|c|c|c|c}
\hline$(n, k)$ & uut $_{s}$ & $(n, k)$ & uut $_{s}$ & $(n, k)$ & Aut $_{s}$ & $(n, k)$ & Aut $_{s}$ \\
\hline$(2,1)$ & 1 & $(7,1)$ & 144 & $(9,3)$ & 8 & $(11,3)$ & 24 \\
$(3,1)$ & 2 & $(7,2)$ & 12 & $(9,4)$ & 4 & $(11,4)$ & 4 \\
$(4,1)$ & 6 & $(7,3)$ & 4 & $(10,1)$ & 14400 & $(11,5)$ & 2 \\
$(4,2)$ & 4 & $(8,1)$ & 720 & $(10,2)$ & 288 & $(12,2)$ & 2880 \\
$(5,1)$ & 12 & $(8,2)$ & 24 & $(10,3)$ & 16 & $(12,3)$ & 48 \\
$(5,2)$ & 4 & $(8,3)$ & 8 & $(10,4)$ & 4 & $(12,4)$ & 4 \\
$(6,1)$ & 36 & $(8,4)$ & 4 & $(10,5)$ & 2 & $(12,5)$ & 2 \\
$(6,2)$ & 8 & $(9,1)$ & 2880 & $(11,1)$ & 86400 & $(12,6)$ & 1 \\
$(6,3)$ & 4 & $(9,2)$ & 72 & $(11,2)$ & 864 & & \\
\hline
\end{tabular}

$\left.k, d^{\perp}\right]$ codes of the inequivalent binary $\operatorname{LCD}[n, k]$ codes. All binary codes in the table can be obtained electronically from http://www . math.is.tohoku.ac.jp/ mharada/LCD2

The smallest possible automorphism group of a binary LCD code is the trivial group (of order 1). It is obvious that the unique binary LCD $[2,1]$ code has trivial automorphism group. We list in Table 2 the smallest value $\mathrm{Aut}_{s}$ among the orders of the automorphism groups of binary LCD codes of lengths up to 12. From the table, we have the following:

Proposition 6. The smallest length $n>2$ for which there is a binary $L C D$ code of length $n$ with trivial automorphism group is 12 .

We remark that the total number of inequivalent binary LCD $[n, k]$ codes for $n=3,4, \ldots, 12$ and $k \leq n / 2$ is 6897,14 of which have trivial automorphism groups. The 14 codes have parameters $[12,6,3]$. As an example, we 
give a binary LCD $[12,6]$ code $B_{12}$ with trivial automorphism group. The code $B_{12}$ has generator matrix $\left(\begin{array}{ll}I_{6} & M_{12}\end{array}\right)$, where

$$
M_{12}=\left(\begin{array}{l}
101011 \\
010110 \\
110100 \\
110001 \\
001101 \\
000011
\end{array}\right) .
$$

Since the smallest possible automorphism group of a binary LCD code is the group of order 1 , there are at least $t_{2}(n, k)=\left\lceil T_{2}(n, k) / n !\right\rceil$ inequivalent binary LCD $[n, k]$ codes. From

$$
\begin{aligned}
& t_{2}(14,1)=1, t_{2}(14,2)=1, t_{2}(14,3)=1, t_{2}(14,4)=18, \\
& t_{2}(14,5)=574, t_{2}(14,6)=4659, t_{2}(14,7)=9282,
\end{aligned}
$$

we have

$$
\sum_{k=1}^{13} t_{2}(14, k)=19790 .
$$

Hence, there are at least 19791 inequivalent binary LCD codes of length 14 . Of course, many binary LCD codes have substantial automorphism groups. Thus, the above might be a poor lower bound. Indeed, there are 30618 inequivalent binary LCD codes of length 13, although we have

$$
\sum_{k=1}^{12} t_{2}(13, k)=2572 .
$$

We continued the above process and completed a classification of binary LCD codes for small dimensions. More precisely, we give a classification of binary LCD $[n, 2]$ codes for $n \leq 30$ and binary LCD $[n, 3]$ codes for $n \leq 25$. In order to save space, we only list in Table 3 the numbers $N$ of all inequivalent binary LCD $[n, k]$ codes for $k=2,3$ and $n \leq 25,30$, respectively.

We end this section with giving the following remark.

Remark 7 . It is a fundamental problem to determine the largest minimum weight $d(n, k)$ among all binary $\mathrm{LCD}[n, k]$ codes. For $1 \leq k \leq n \leq 12$, the values $d(n, k)$ were determined in [5]. For $1 \leq k \leq n$ and $n=13,14,15,16$, the values $d(n, k)$ were determined in [8]. Also, a classification of binary LCD $[n, k]$ codes having the minimum weight $d(n, k)$ was given in [8] for $1 \leq k \leq n \leq 16$. 
Table 3: Classification of binary LCD codes of dimensions 2 and 3

\begin{tabular}{c|c|c|c|c|c|c|c}
\hline$(n, k)$ & $N$ & $(n, k)$ & $N$ & $(n, k)$ & $N$ & $(n, k)$ & $N$ \\
\hline$(14,2)$ & 66 & $(19,2)$ & 136 & $(24,2)$ & 270 & $(29,2)$ & 431 \\
$(15,2)$ & 73 & $(20,2)$ & 166 & $(25,2)$ & 286 & $(30,2)$ & 495 \\
$(16,2)$ & 93 & $(21,2)$ & 178 & $(26,2)$ & 335 & & \\
$(17,2)$ & 101 & $(22,2)$ & 214 & $(27,2)$ & 354 & & \\
$(18,2)$ & 126 & $(23,2)$ & 228 & $(28,2)$ & 410 & & \\
\hline$(14,3)$ & 380 & $(17,3)$ & 1120 & $(20,3)$ & 2648 & $(23,3)$ & 6074 \\
$(15,3)$ & 576 & $(18,3)$ & 1468 & $(21,3)$ & 3608 & $(24,3)$ & 7580 \\
$(16,3)$ & 772 & $(19,3)$ & 2058 & $(22,3)$ & 4568 & $(25,3)$ & 9870 \\
\hline
\end{tabular}

\section{Classification of ternary LCD codes of lengths up to 10}

By an approach is similar to that used in the previous section, we completed a classification of ternary $\operatorname{LCD}[n, k]$ codes for $2 \leq k \leq n / 2 \leq 10 / 2$. For $1 \leq n \leq 7$, we found all distinct ternary LCD $[n, k]$ codes, which must be checked to achieve a complete classification. By determining the equivalence or inequivalence for a given pair of ternary codes, we obtained the set $\mathcal{C}_{n, k}$. The mass formula (3) shows that there is no other ternary LCD $[n, k]$ code. For $n=8,9$ and 10, the mass formula (3) was used to complete the classification, due to the computational complexity. For ternary LCD codes, the following method was employed. To test equivalence of ternary codes by a program in the language $\mathrm{C}$, we used the algorithm given in [9, Section 7.3.3] as follows. For a ternary $[n, k]$ code $C$, define the digraph $\Gamma(C)$ with vertex set $C \cup\left(\{1,2, \ldots, n\} \times\left(\mathbb{F}_{3}-\{0\}\right)\right)$ and arc set $\left\{\left(c,\left(j, c_{j}\right)\right) \mid c=\left(c_{1}, \ldots, c_{n}\right) \in\right.$ $C, 1 \leq j \leq n\} \cup\left\{((j, y),(j, 2 y)) \mid 1 \leq j \leq n, y \in \mathbb{F}_{3}-\{0\}\right\}$. Then, two ternary $[n, k]$ codes $C$ and $C^{\prime}$ are equivalent if and only if $\Gamma(C)$ and $\Gamma\left(C^{\prime}\right)$ are isomorphic. We used NAUTY [12] for digraph isomorphism testing. The automorphism group $\operatorname{Aut}(C)$ is isomorphic to the automorphism group of $\Gamma(C)$. This calculation was also done by using NAUTY [12].

As a check, in order to verify that $\mathcal{C}_{n, k}$ contains no pair of equivalent ternary LCD $[n, k]$ codes and that $\sum_{C \in \mathcal{C}_{n, k}} \frac{2^{n} n !}{\operatorname{Aut}(C) \mid}=T_{3}(n, k)$, we employed the following method obtained by applying the method given in [10, Section 2]. Let $C$ be a ternary $[n, k]$ code. We expand each codeword of $C$ into 
a binary vector of length $2 n$ by mapping the elements 0,1 and 2 of $\mathbb{F}_{3}$ to the binary vectors $(0,0),(0,1)$ and $(1,0)$, respectively. If there is a positive integer $t$ such that the codewords of weight $t$ generate $C$, then we have an $A_{t} \times 2 n$ binary matrix $M(C)$ composed of the binary vectors obtained from the $A_{t}$ codewords of weight $t$ in $C$. If there is no positive integer $t$ such that the codewords of weight $t$ generate $C$, then by considering all codewords of $C$, we have a $3^{k} \times 2 n$ binary matrix $M(C)$. Then, from $M(C)$, we have an incidence structure $\mathcal{D}(C)$ having $2 n$ points. This calculation was done by using the MAgma function IncidenceStructure. If $C$ and $C^{\prime}$ are equivalent ternary codes, then $\mathcal{D}(C)$ and $\mathcal{D}\left(C^{\prime}\right)$ are isomorphic. By the Magma function IsIsomorphic, we verified that all incidence structures $\mathcal{D}(C)$ are non-isomorphic. The automorphism group $\operatorname{Aut}(C)$ is isomorphic to the stabilizer of $\{\{1,2\},\{3,4\}, \ldots,\{2 n-1,2 n\}\}$ inside of the automorphism group of the incidence structure $\mathcal{D}(C)$. This calculation was done by using the MAGMA functions AutomorphismGroup and Stabilizer.

We list in Table 4 the numbers $N$ of the inequivalent ternary LCD $[n, k]$ codes and the numbers $N_{d}$ of the inequivalent ternary LCD $[n, k, d]$ codes for $2 \leq k \leq n / 2$ and $n \leq 10$. We also list the numbers $N_{d^{\perp}}$ of the dual $[n, n-$ $\left.k, d^{\perp}\right]$ codes of the inequivalent ternary LCD $[n, k]$ codes. All ternary codes in the table can be obtained electronically from http://www . math.is.tohoku .ac.jp/ mharada/LCD3

The smallest possible automorphism group of a ternary LCD code is the

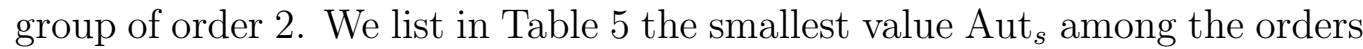
of the automorphism groups of ternary $\operatorname{LCD}[n, k]$ codes for $n \leq 8$. From the table, we have the following:

Proposition 8. The smallest length $n$ for which there is a ternary LCD code of length $n$ with automorphism group of order 2 is 8 .

We remark that the total number of inequivalent ternary LCD $[n, k]$ codes for $n=2,3, \ldots, 8$ and $k \leq n / 2$ is 336 , one of which has automorphism group of order 2. The code has parameters $[8,4,3]$. As an example, we give a ternary LCD $[8,4]$ code $C_{8}$ with automorphism group of order 2 . The code $C_{8}$ has generator matrix $\left(\begin{array}{ll}I_{4} & M_{8}\end{array}\right)$, where

$$
M_{8}=\left(\begin{array}{l}
2001 \\
2212 \\
1100 \\
1012
\end{array}\right)
$$


Table 4: Classification of ternary LCD codes

\begin{tabular}{c|c|ccccccc|cccc}
\hline$(n, k)$ & $N$ & $N_{1}$ & $N_{2}$ & $N_{3}$ & $N_{4}$ & $N_{5}$ & $N_{6}$ & $N_{7}$ & $N_{1^{\perp}}$ & $N_{2^{\perp}}$ & $N_{3^{\perp}}$ & $N_{4}$ \\
\hline$(4,2)$ & 4 & 2 & 2 & & & & & & 2 & 2 & & \\
\hline$(5,2)$ & 7 & 3 & 3 & 1 & & & & & 4 & 3 & & \\
\hline$(6,2)$ & 11 & 4 & 4 & 2 & 1 & & & & 7 & 4 & & \\
$(6,3)$ & 17 & 7 & 8 & 2 & & & & & 7 & 8 & 2 & \\
\hline$(7,2)$ & 16 & 4 & 6 & 3 & 3 & & & & 11 & 5 & & \\
$(7,3)$ & 36 & 11 & 17 & 7 & 1 & & & & 17 & 17 & 2 & \\
\hline$(8,2)$ & 24 & 5 & 7 & 4 & 6 & 2 & & & 16 & 8 & & \\
$(8,3)$ & 74 & 16 & 31 & 19 & 8 & & & & 36 & 37 & 1 & \\
$(8,4)$ & 121 & 36 & 64 & 19 & 2 & & & & 36 & 64 & 19 & 2 \\
\hline$(9,2)$ & 33 & 6 & 8 & 5 & 9 & 4 & 1 & & 24 & 9 & & \\
$(9,3)$ & 149 & 24 & 51 & 40 & 31 & 3 & & & 74 & 74 & 1 & \\
$(9,4)$ & 379 & 74 & 178 & 105 & 22 & & & & 121 & 218 & 40 & \\
\hline$(10,2)$ & 45 & 6 & 10 & 6 & 11 & 8 & 3 & 1 & 33 & 12 & & \\
$(10,3)$ & 290 & 33 & 80 & 70 & 84 & 22 & 1 & & 149 & 140 & 1 & \\
$(10,4)$ & 1293 & 149 & 458 & 431 & 249 & 6 & & & 379 & 821 & 93 & \\
$(10,5)$ & 2318 & 379 & 1209 & 665 & 65 & & & & 379 & 1209 & 665 & 65 \\
\hline
\end{tabular}

Since the smallest possible automorphism group of a ternary LCD code is the group of order 2 , there are at least $t_{3}(n, k)=\left\lceil T_{3}(n, k) /\left(2^{n-1} n !\right)\right\rceil$ inequivalent ternary LCD $[n, k]$ codes. From

$$
\begin{aligned}
& t_{3}(11,1)=1, t_{3}(11,2)=1, t_{3}(11,3)=4 \\
& t_{3}(11,4)=319, t_{3}(11,5)=2869
\end{aligned}
$$

Table 5: Smallest automorphism groups

\begin{tabular}{c|c|c|c|c|c|c|c}
\hline$(n, k)$ & $\mathrm{Aut}_{s}$ & $(n, k)$ & $\mathrm{Aut}_{s}$ & $(n, k)$ & $\mathrm{Aut}_{s}$ & $(n, k)$ & $\mathrm{Aut}_{s}$ \\
\hline$(2,1)$ & 4 & $(5,1)$ & 96 & $(6,3)$ & 8 & $(8,1)$ & 11520 \\
$(3,1)$ & 8 & $(5,2)$ & 16 & $(7,1)$ & 1920 & $(8,2)$ & 96 \\
$(4,1)$ & 32 & $(6,1)$ & 384 & $(7,2)$ & 48 & $(8,3)$ & 8 \\
$(4,2)$ & 8 & $(6,2)$ & 24 & $(7,3)$ & 8 & $(8,4)$ & 2 \\
\hline
\end{tabular}


we have

$$
\sum_{k=1}^{10} t_{3}(11, k)=6388
$$

Hence, there are at least 6389 inequivalent ternary LCD codes of length 11. Of course, many ternary LCD codes have substantial automorphism groups. Thus, the above might be a poor lower bound. Indeed, there are 5588 inequivalent ternary LCD codes of length 10, although we have

$$
\sum_{k=1}^{9} t_{3}(10, k)=447 \text {. }
$$

Similar to Remark 7, we end this section with giving the following remark. Remark 9. Let $d_{3}(n, k)$ and $d_{3}^{\text {all }}(n, k)$ denote the largest minimum weight among all ternary LCD $[n, k]$ codes and among all ternary $[n, k]$ codes, respectively. Suppose that $2 \leq k \leq n-2$ and $4 \leq n \leq 10$. From Table 4 , we have

$$
d_{3}(n, k)= \begin{cases}d_{3}^{\text {all }}(n, k)-1 & \text { if }(n, k) \in S \\ d_{3}^{\text {all }}(n, k) & \text { otherwise }\end{cases}
$$

where

$$
S=\{(4,2),(7,2),(8,2),(8,3),(9,3),(9,4),(9,5),(10,4),(10,5),(10,6)\} .
$$

Note that the values $d_{3}^{\text {all }}(n, k)$ can be found in [7].

Acknowledgment. This work was supported by JSPS KAKENHI Grant Number $15 \mathrm{H} 03633$.

\section{References}

[1] R. Baart, T. Boothby, J. Cramwinckel, J. Fields, D. Joyner, R. Miller, E. Minkes, E. Roijackers, L. Ruscio and C. Tjhai, GAP package GUAVA, Version 3.1.3; 2016, Available online at http://www.gap-system.org/Packages/guava.html.

[2] W. Bosma, J. Cannon and C. Playoust, The Magma algebra system I: The user language, J. Symbolic Comput. 24 (1997), 235-265. 
[3] C. Carlet, S. Mesnager, C. Tang and Y. Qi, New characterization and parametrization of LCD codes, arXiv:1709.03217.

[4] C. Carlet, S. Mesnager, C. Tang, Y. Qi and R. Pellikaan, Linear codes over $\mathbb{F}_{q}$ are equivalent to LCD codes for $q>3$, IEEE Trans. Inform. Theory 64 (2018), 3010-3017.

[5] L. Galvez, J.-L. Kim, N. Lee, Y.G. Roe and B.-S. Won, Some bounds on binary LCD codes, Cryptogr. Commun. 10 (2018), 719-728.

[6] The GAP Group, GAP-Groups, Algorithms, and Programming, Version 4.8.10; 2018, Available online at http://www.gap-system.org.

[7] M. Grassl, Code tables: Bounds on the parameters of various types of codes, Available online at http://www.codetables.de/, Accessed on 2018-03-30.

[8] M. Harada and K. Saito, Binary linear complementary dual codes, arXiv: 1802.06985 .

[9] P. Kaski and P.R.J. Östergård, Classification Algorithms for Codes and Designs, Springer, Berlin, 2006.

[10] C.W.H. Lam, L. Thiel and A. Pautasso, On ternary codes generated by Hadamard matrices of order 24, Congr. Numer. 89 (1992), 7-14.

[11] J.L. Massey, Linear codes with complementary duals, Discrete Math. 106/107 (1992), 337-342.

[12] B.D. McKay and A. Piperno, nauty and Traces User's Guide (Version 2.6), Available online at http://users. cecs. anu .edu. au/ bdm/nauty/nug26.pdf. 
Table 6: Classification of binary LCD codes

\begin{tabular}{|c|c|c|c|c|c|c|c|c|c|c|c|c|c|}
\hline$(n, k)$ & $N$ & $N_{1}$ & $N_{2}$ & $N_{3}$ & $N_{4}$ & $N_{5}$ & $N_{6}$ & $N_{7}$ & $N_{8}$ & $N_{1 \perp}$ & $N_{2 \perp}$ & $N_{3^{\perp}}$ & $N_{4 \perp}$ \\
\hline$(4,2)$ & 4 & 2 & 2 & & & & & & & 2 & 2 & & \\
\hline$(5,2)$ & 5 & 2 & 3 & & & & & & & 4 & 1 & & \\
\hline$(6,2)$ & 9 & 3 & 4 & 2 & & & & & & 5 & 4 & & \\
\hline$(6,3)$ & 8 & 5 & 3 & & & & & & & 5 & 3 & & \\
\hline$(7,2)$ & 11 & 3 & 5 & 2 & 1 & & & & & 9 & 2 & & \\
\hline$(7,3)$ & 17 & 9 & 7 & 1 & & & & & & 8 & 9 & & \\
\hline$(8,2)$ & 17 & 4 & 6 & 4 & 2 & 1 & & & & 11 & 6 & & \\
\hline$(8,3)$ & 26 & 11 & 12 & 3 & & & & & & 17 & 9 & & \\
\hline$(8,4)$ & 42 & 17 & 24 & 1 & & & & & & 17 & 24 & 1 & \\
\hline$(9,2)$ & 20 & 4 & 7 & 4 & 3 & 1 & 1 & & & 17 & 3 & & \\
\hline$(9,3)$ & 49 & 17 & 20 & 11 & 1 & & & & & 26 & 23 & & \\
\hline$(9,4)$ & 81 & 26 & 49 & 5 & 1 & & & & & 42 & 37 & 2 & \\
\hline$(10,2)$ & 29 & 5 & 8 & 6 & 4 & 4 & 2 & & & 20 & 9 & & \\
\hline$(10,3)$ & 72 & 20 & 29 & 18 & 4 & 1 & & & & 49 & 23 & & \\
\hline$(10,4)$ & 186 & 49 & 109 & 23 & 5 & & & & & 81 & 103 & 2 & \\
\hline$(10,5)$ & 204 & 81 & 112 & 11 & & & & & & 81 & 112 & 11 & \\
\hline$(11,2)$ & 33 & 5 & 9 & 6 & 5 & 4 & 4 & & & 29 & 4 & & \\
\hline$(11,3)$ & 123 & 29 & 42 & 35 & 11 & 6 & & & & 72 & 51 & & \\
\hline$(11,4)$ & 348 & 72 & 195 & 61 & 20 & & & & & 186 & 161 & 1 & \\
\hline$(11,5)$ & 606 & 186 & 350 & 66 & 4 & & & & & 204 & 386 & 15 & 1 \\
\hline$(12,2)$ & 45 & 6 & 10 & 8 & 6 & 7 & 6 & 2 & & 33 & 12 & & \\
\hline$(12,3)$ & 174 & 33 & 56 & 48 & 22 & 14 & 1 & & & 123 & 51 & & \\
\hline$(12,4)$ & 744 & 123 & 369 & 170 & 76 & 6 & & & & 348 & 396 & & \\
\hline$(12,5)$ & 1584 & 348 & 909 & 290 & 37 & & & & & 606 & 956 & 22 & \\
\hline$(12,6)$ & 2426 & 606 & 1622 & 187 & 11 & & & & & 606 & 1622 & 187 & 11 \\
\hline$(13,2)$ & 50 & 6 & 11 & 8 & 7 & 7 & 8 & 2 & 1 & 45 & 5 & & \\
\hline$(13,3)$ & 277 & 45 & 75 & 77 & 39 & 35 & 6 & & & 174 & 103 & & \\
\hline$(13,4)$ & 1363 & 174 & 598 & 341 & 217 & 31 & 2 & & & 744 & 619 & & \\
\hline$(13,5)$ & 4576 & 744 & 2354 & 1178 & 295 & 5 & & & & 1584 & 2965 & 27 & \\
\hline$(13,6)$ & 9036 & 1584 & 5900 & 1406 & 146 & & & & & 2426 & 6086 & 520 & 4 \\
\hline
\end{tabular}

